\title{
Evolution of cardiac computerized tomography in India
}

\author{
Hemant B. Telkar \\ Department of Radiology, Jupiter Heart Scan, Mumbai, India
}

\begin{abstract}
Correspondence: Dr. Hemant B Telkar, Jupiter Heart Scan, Garden View, 756 Mancherji Joshi Marg, Parsi Colony, Mumbai - 400014, India. E-mail: telkar@vsnl.com
\end{abstract}

I find myself immensely lucky to have been the first person in India to be involved with a '4-slice' MDCT scanner, for cardiac imaging, from the latter half of 2001.

Being a radiologist, I had forgotten the anatomy of the heart. I had to go back to Gray's textbook of anatomy and then start looking at cardiac images. Data from the 4-slice scanner was quite informative. It was just as if moving body parts were 'frozen in time' and displayed for analysis.

I remember the first time I saw images of the coronary arteries in Brazil, where I had my formal training and my first question was 'Is this from a cadaveric heart?' In that era, just the visualization and identification of the coronary arteries was thrilling and challenging. Challenging, because there were only a couple of international papers from Germany written on how to perform cardiac computerized tomography (CT) scans. I had to work with my judgment to work out a reasonable protocol for the Indian setting.

The two biggest challenges during the '4-slice' era were getting the heart rate (HR) to around $50 / 55 \mathrm{bpm}$ and making patients hold their breath for about 40 seconds.

In spite of these challenges, in a reasonably good number of patients, we were able to obtain good data sets, albeit after heavily beta-blocking the patients.

The 4-slice technology had a temporal resolution of 250 ms and a rotation time of $500 \mathrm{~ms}$. Our major drawback however, was the failure to recognize artifact-induced 'pseudo-stenosis', mainly due to lack of experience. This led to many false-positive results and hence the reliability of the modality was a big question mark in the minds of the referring physicians and cardiologists.

16-slice MDCT scanner became available in December 2003. This was a major technology break-through. The 16-row detector scanner resulted in good datasets with a reasonable HR of about $60-65 \mathrm{bpm}$. The breath-hold time decreased to
15-20 seconds and the temporal resolution improved from $250 \mathrm{~ms}$ to $92 \mathrm{~ms}$. We were consistently able to accurately localize and describe the lesions, but estimation of the grade of stenosis was not very accurate. The issue of motion artifact decreased to some extent but evaluation of heavily calcified arteries was still an issue.

Imaging of post-bypass grafts became much easier on the 16-slice scanner. Evaluating stents was still an issue in many of our patients. Obese patients were a problem due to the low signal to noise ratio. 16-slice technology made further progress when the rotation time was reduced from $420 \mathrm{~ms}$ to $370 \mathrm{~ms}$, which helped improve the temporal resolution. High mAs became possible on this scanner due to the development of new tube technology. This allowed us to successfully scan obese patients, but the issue of radiation came to the fore. Though new software allows us to decrease the radiation by scanning mainly in the mid-diastolic phase, this has its limitations.

A few international papers were published on 16-slice technology to evaluate the specificity, sensitivity and the negative and positive predictive values. Virtually all papers showed high negative predictive values and an increasing number of assessable segments. This allowed referring clinicians to consider non-invasive cardiac CT in patients with atypical chest pain and in those with a low pretest probability of coronary artery disease (CAD), in the setting of "to rule out CAD".

All through these years, unlike in other parts of the world, calcium scoring for diagnostic risk stratification did not take off in India. This is mainly because we do not have a system or platform to use any risk stratification system in our health care pyramid on a mass-scale.

As more and more 16-slice CT scanners from different vendors were being installed in our country, the overall awareness increased. International conferences started addressing the technology seriously and many physicians 
started saying 'Yes, there is now a technology which probably I could use', though most were still not sure of when and how to use the technology.

I started interacting with other 16-slice CT users who were performing cardiac CT scans. Among many incidents, one stands out. Dr. Kulkarni reported a case of a patient with RCA bridging. ${ }^{[1]}$ When he first generated a patient report describing the intra-myocardial course of the RCA, his cardiologist laughed at him, saying that no such entity existed.

The first 64-slice scanner was launched in 2005 and our 16-slice CT scanner was soon upgraded thereafter, as well. In conferences as well as in the lay press, "64-slice" caught the imagination of both, the physicians and the general public and this has led to specific demand-based referral for cardiac CT angiography on a 64-slice scanner.

With the 64-slice CT scanner, we also moved beyond coronary artery evaluation and started looking at function; especially ejection fraction and wall motion, as well as started answering questions in pediatric cardiology.

Currently in India, we have more than twenty 64-slice scanners. This increasing number has helped to create awareness and thus a wider base is now available for the use of this modality.

As we move ahead, we now have new software that allows us to quantify stenoses with accuracy rates, comparable to quantitative coronary angiography. The first dual source CT scanners have already been installed and it is claimed that heart-rate control is no longer required and visualization of the lumen through heavily calcified plaques as well as within stents, has improved considerably.

I am confident and sure that we, the Indian radiologists have the resources and mind-sets to be on the same platform as the international fraternity and will develop and take this new specialty of cardiac-imaging ahead.

This guest issue is partly aimed at demonstrating our experience with cardiac CT for the benefit of our colleagues in India and abroad.

\section{Reference}

1. Kulkarni M, Sodani A, Rosita, Puranik C, Sullere S, Saha B. Right myocardial bridge on CT coronary angiography. J Assoc Physicians India 2004;52:661-2.

\section{Announcement}

\section{Free access to the Cochrane Library for everyone in India}

Anyone in India with access to the Internet now has complementary access to reliable, up-to-date evidence on health care interventions from The Cochrane Library, thanks to sponsorship provided by the Indian Council of Medical Research (ICMR) that recently signed a three-year contract for a national subscription with the publishers, John Wiley \& Sons.

The Cochrane Library (available at www.thecochranelibrary.com) is considered by many to be the single most reliable source for evidence on the effects of health care interventions. It includes seven databases that are updated quarterly, four of which are the efforts of the 15,000 international contributors of the Cochrane Collaboration (www.cochrane.org).

The Cochrane Database of Systematic Reviews currently contains 4655 regularly-updated systematic reviews and protocols of reviews in preparation

The Cochrane Controlled Trials Register currently contains references, mostly with abstracts, of more than 48,900 controlled clinical trials- easily the largest collection of such trials in the world

The Cochrane Database of Methodology Reviews contains 22 systematic reviews of the science of reviewing evidence The Cochrane Methodology Register contains the bibliography of 9048 articles that could be relevant to anyone preparing systematic reviews.

The three other databases in The Cochrane Library include the:

Database of Abstracts of Reviews of Effectiveness, summaries of 5931 systematic reviews published elsewhere and quality appraised by the UK National Health Service (NHS) Centre for Reviews and Dissemination

Health Technology Assessment Database that contains details of 6358 completed and ongoing health technology assessments

NHS Economic Evaluation Database that contains 20, 292 abstracts of quality assessed economic evaluations from around the world.

Also available is information about the Cochrane Collaboration. One can search for interventions or health conditions across all these databases using free text terms or medical subject headings $(\mathrm{MeSH})$.

From 29 January 2007 the Cochrane Library is freely available to all residents of India with Internet access thanks to funding from the Indian Council of Medical Research (ICMR) (www.ICMR.nic.in), and work of the South Asia Cochrane Network (www.cochrane-sacn.org). 